\title{
Caracterização físico-química de frutos de genótipos de aceroleira (Malpighia emarginata D.C.)
}

\author{
Physicochemical characterization of fruits from genotypes of acerola tree (Malpighia emarginata D.C.)
}

\author{
Maria Inês Sucupira MACIEL ${ }^{1 \star}$, Enayde MÉLO ${ }^{1}$, Vera LIMA ${ }^{1}$, Kelvina Araújo SOUZA ${ }^{1}$, Wedja SILVA²
}

\begin{abstract}
Resumo
No Brasil, a aceroleira, decorrente principalmente da propagação por sementes, tem dado origem a plantios comerciais cujos frutos apresentam parâmetros de qualidade diferenciados. Características físico-químicas de frutos de 18 genótipos de aceroleira (Malpighia emarginata DC.) do Banco Ativo de Germoplasma da Universidade Federal Rural de Pernambuco - UFRPE foram avaliados. O rendimento em polpa variou de 41,06\% (PL 40) a 72,54\% (PL 43) e, com exceção do genótipo PL 37, os demais apresentaram frutos com teores de ácido ascórbico superiores a $1000 \mathrm{mg} .100 \mathrm{~g} \mathrm{~g}^{-1}$. O genótipo PL 39 destacou-se por apresentar o maior teor de ácido ascórbico $(1667 \mathrm{mg} .100 \mathrm{~g}$-1), SST e flavonóis $\left(15,04 \mathrm{mg} .100 \mathrm{~g}^{-1}\right)$, além de elevado teor de antocianinas, sendo, portanto, o mais promissor. Os frutos do genótipo PL 34 revelaram o maior valor de SST/ATT, indicando ser o mais doce.

Palavras-chave: acerola; ácido ascórbico; rendimento; antocianinas; flavonóis.
\end{abstract}

\begin{abstract}
In Brazil, acerola tree commercial orchards yield fruits with different quality attributes since their propagation is by seeds. The physicochemical characteristics of 18 genotypes of acerola tree (Malpighia emarginata DC.) from the Active Bank of Germoplasm of UFRPE were evaluated. The pulp efficiency ranged from 41.06 (PL 40) to 72.54\% (PL 43). Except for the genotype PL 37, all others showed ascorbic acid content higher than 1,000 mg.100 g- The genotype PL 39 showed the highest content of ascorbic acid $\left(1,667 \mathrm{mg}^{-1} 100 \mathrm{~g}^{-1}\right)$, TSS and flavonols (15.04 mg. $100 \mathrm{~g}^{-1}$ ) and a high value for anthocyanins. Therefore, it can be said it is the promising genotype. The fruits from genotype PL 34 showed the highest value for the TSS/TTA; they were the sweetest.

Keywords: acerola; ascorbic acid; pulp efficiency; anthocyanins; flavonols.
\end{abstract}

\section{Introdução}

A acerola (Malpighia emarginata D.C.) é uma fruta que tem se destacado por ser uma excelente fonte natural de vitamina $\mathrm{C}$. Também conhecida como cereja-das-antilhas, tem como região de origem as Antilhas, Norte da América do Sul e América Central (MATSUURA; ROLIM, 2002). De acordo com Lopes e Paiva (2002), a acerola foi introduzida no Estado de Pernambuco em 1956 através de algumas sementes trazidas de Porto Rico pela professora Maria Celene Cardoso de Almeida, da Universidade Federal Rural de Pernambuco.

O Brasil é um dos maiores produtores mundiais de acerola, destacando-se a região Nordeste com uma produção de aproximadamente 22.500 toneladas de frutos (UNIDADES DE PRODUTOS PARA FRUTICULTURA, 2006).

Aliado ao alto teor de vitamina $\mathrm{C}$, este fruto ainda é fonte de antocianinas (MUSSER et al., 2004) e de carotenoides (LIMA et al., 2005), compostos que exercem efeitos benéficos à saúde humana uma vez que possuem reconhecida ação antioxidante (KÄHKÖNEN; HEINONEN, 2003; KRINSKY, 1989).

Embora seja consumida in natura, a acerola é mais utilizada na produção de néctar, polpa congelada, suco pasteurizado engarrafado, geleia, entre outros ou ainda no enriquecimento de vitamina $\mathrm{C}$ em néctares ou sucos de frutas pobres nessa vitamina (MARINO NETTO, 1986; MATSUURA et al., 2001; AGOSTINI-COSTA; ABREU; ROSSETI, 2003; YAMASHITA et al., 2003). Sendo assim, o cultivo da acerola torna-se altamente promissor, apresentando boas perspectivas para o mercado fruticultor.

No Brasil, grande parte dos pomares comerciais foi formada a partir de propagação assexuada, o que resultou em uma ampla variabilidade genética (MACIEL et al., 1999). Segundo Carpentieri-Pípolo et al. (2002), é possível observar nesses pomares plantas com hábito de crescimento diferenciado e produção de frutas quantitativa e qualitativamente heterogênea. Esta constatação, associada à importância econômica da acerola para o Estado de Pernambuco, motivou a instalação de um Banco Ativo de Germoplasma de acerola. Dos 42 genótipos instalados, 12 já foram avaliados quanto às características físico-químicas (MUSSER et al., 2004) e, com a finalidade de dar continuidade à avaliação dos demais, este trabalho teve como objetivo determinar as características físico-químicas dos frutos de mais 18 genótipos deste mesmo Banco.

Departamento de Ciências Domésticas, Universidade Federal Rural de Pernambuco - UFRPE, Av. Dois Irmãos, s/n, Dois Irmãos, CEP 52171-900, Recife - PE, Brasil,

E-mail:m.ines@dcd.ufrpe.br

2 Departamento de Tecnologia de Alimentos, Universidade Federal do Ceará - UFC, Fortaleza - CE, Brasil

${ }^{*}$ A quem a correspondência deve ser enviada 


\section{Material e métodos}

Foram utilizadas acerolas de 18 genótipos cultivados no Banco Ativo de Germoplasma da Universidade Federal Rural de Pernambuco, instalado na Estação Experimental de Canade-açúcar do Carpina, município de Carpina - PE, com latitude de $7^{\circ} 51^{\prime} 04^{\prime}$, longitude $35^{\circ} 14^{\prime} 27^{\prime \prime} \mathrm{W}$, a $178 \mathrm{~m}$ de altitude, predominando o tipo climático "AS" tropical chuvoso com verão seco, segundo a classificação de Köppen. Durante as safras de 20032004, frutos maduros, colhidos pela manhã, foram transportados em caixas isotérmicas até o Laboratório de Análise Físico-química de Alimentos do Departamento de Ciências Domésticas/UFRPE, onde foram analisados quanto às características: rendimento do suco (\%) extraído em centrífuga doméstica, obtido pela diferença entre o peso dos frutos e o peso do seu resíduo; ácido ascórbico determinado pelo método titulométrico, utilizando 2,6 diclorofenol indofenol; sólidos solúveis totais, acidez total titulável e $\mathrm{pH}$, segundo metodologia descrita pela AOAC (ASSOCIATION..., 1990); e antocianinas e flavonóis totais de acordo com o método proposto por Lees e Francis (1972).

O delineamento experimental foi inteiramente casualizado com três repetições (utilizando três árvores de cada genótipo). Os resultados foram submetidos à análise de variância e teste de Tukey a 5\% de probabilidade, utilizando o programa "STATISTICA" na versão 6.0, para Windows.

O estudo das diferenças dos genótipos de acerola foi feito através da utilização da Análise de Componentes Principais (ACP), segundo o programa STAT-ITCF. A ACP permite a construção de planos fatoriais, nos quais os eixos são combinações lineares das variáveis iniciais (descritores) e a visualização das relações entre essas variáveis e os produtos (genótipos) (FOUCART, 1982).

\section{Resultados e discussão}

Os teores de ácido ascórbico variaram de 750 a $1.678 \mathrm{mg} .100 \mathrm{~g}^{-1}$ de polpa (Tabela 1). Os frutos do genótipo PL 39 apresentaram o mais elevado valor desta vitamina, diferindo significativamente dos frutos dos genótipos PL 34, PL 36, PL 37, PL 38 e PL 40. Na caracterização química de três matrizes de acerola, França e Narain (2003) relataram, para frutos maduros, valores de 940 a 2000 mg.100 g g $^{-1}$ de polpa. Gonzaga Neto, Mattuz e Santos (1999) encontraram valores entre 799 e $2444 \mathrm{mg}$ de ácido ascórbico.100 $\mathrm{g}^{-1}$ de polpa em clones de aceroleira na região do submédio São Francisco. Musser et al. (2004), avaliando as características físico-químicas dos primeiros 12 genótipos do Banco Ativo de Germoplasma da UFRPE, relataram que a variação média do teor de ácido ascórbico foi de 1067 a 1846 mg.100 g ${ }^{-1}$ de polpa. Segundo Nogueira et al. (2002), acerolas maduras, provenientes do pomar comercial Acerolândia em Paudalho - PE, apresentaram teores de ácido ascórbico de 865,80 a 1797,80 mg. $100 \mathrm{~mL}^{-1}$ de suco. Enquanto, Mezadri et al. (2008), encontraram valores de ácido ascórbico entre 632 a $920 \mathrm{mg} .100 \mathrm{~g}^{-1}$ de polpa em acerolas maduras do Distrito de Itajaí - SC.

$\mathrm{O}$ teor de vitamina $\mathrm{C}$ na acerola pode ser influenciado por vários fatores como a localização geográfica, práticas de cultivo, regime pluvial, exposição à luz do sol, características genéticas e, principalmente, o estádio de maturação em que os frutos se encontram. Diversos pesquisadores constataram que o conteúdo desta vitamina diminui durante o amadurecimento do fruto (NOGUEIRA et al., 2002; ASSIS; LIMA; OLIVEIRA, 2001; VENDRAMINI; TRUGO, 2000; ALVES; CHITARRA, A. B.; CHITARRA, M. I. F., 1995).

Tabela 1. Análises físico-químicas em frutos de 18 genótipos de aceroleiras do Banco Ativo de Germoplasma da UFRPE.

\begin{tabular}{|c|c|c|c|c|c|c|}
\hline $\begin{array}{l}\text { Genótipos } \\
\text { (códigos) }\end{array}$ & $\begin{array}{c}\text { AA } \\
\left(\mathrm{mg} .100 \mathrm{~g}^{-1}\right)\end{array}$ & $\begin{array}{c}\text { ATT } \\
\text { (g de ácido málico.100 g-1) }\end{array}$ & $\begin{array}{c}\mathrm{SST} \\
\left({ }^{\circ} \text { Brix }\right)\end{array}$ & SST/ATT & $\mathrm{pH}$ & $\begin{array}{c}\mathrm{R} \\
(\%)\end{array}$ \\
\hline PL 26 & $1429^{\mathrm{ab}} \pm 173,47$ & $1,56^{\mathrm{ab}} \pm 0,11$ & $7,13^{\mathrm{b}} \pm 0,23$ & $4,57^{\mathrm{ab}} \pm 0,21$ & $3,3^{\mathrm{a}} \pm 0,0$ & $57,57^{\text {defg }} \pm 3,08$ \\
\hline PL 28 & $1257^{\mathrm{abc}} \pm 62,44$ & $1,56^{\mathrm{ab}} \pm 0,11$ & $7,96^{\mathrm{b}} \pm 0,05$ & $5,12^{\mathrm{ab}} \pm 0,39$ & $3,2^{\mathrm{a}} \pm 0,05$ & $53,17^{\mathrm{g}} \pm 1,13$ \\
\hline PL 29 & $1429^{\mathrm{ab}} \pm 95,38$ & $1,97^{\mathrm{a}} \pm 0,06$ & $8,13^{\mathrm{b}} \pm 0,32$ & $4,12^{\mathrm{b}} \pm 0,12$ & $3,1^{\mathrm{a}} \pm 0,05$ & $60,97^{\text {bcd }} \pm 1,77$ \\
\hline PL 31 & $1166^{\mathrm{abc}} \pm 89,46$ & $1,59^{\mathrm{ab}} \pm 0,06$ & $8,33^{\mathrm{b}} \pm 1,15$ & $5,23^{\mathrm{ab}} \pm 0,90$ & $3,2^{\mathrm{a}} \pm 0,11$ & $59,73^{\mathrm{cdfg}_{ \pm}}+1,64$ \\
\hline PL 32 & $1174^{\mathrm{abc}} \pm 265,32$ & $1,41^{\mathrm{ab}} \pm 0,12$ & $8,00^{\mathrm{b}} \pm 1,00$ & $5,65^{\mathrm{ab}} \pm 0,38$ & $3,0^{\mathrm{a}} \pm 0,15$ & $55,36^{\mathrm{fg}} \pm 0,44$ \\
\hline PL 33 & $1117^{\mathrm{abc}} \pm 65,65$ & $1,30^{\mathrm{b}} \pm 0,06$ & $7,66^{\mathrm{b}} \pm 0,57$ & $5,91^{\mathrm{ab}} \pm 0,66$ & $3,1^{\mathrm{a}} \pm 0,05$ & $55,27^{\mathrm{fg}} \pm 4,62$ \\
\hline PL 34 & $1043^{b c} \pm 305,51$ & $1,22^{b} \pm 0,19$ & $8,66^{\mathrm{b}} \pm 1,60$ & $7,06^{\mathrm{a}} \pm 0,44$ & $3,3^{\mathrm{a}} \pm 0,20$ & $56,36^{\mathrm{efg}} \pm 2,11$ \\
\hline PL 35 & $1438^{\mathrm{ab}} \pm 404,79$ & $1,33^{\mathrm{b}} \pm 0,19$ & $8,06^{\mathrm{b}} \pm 1,50$ & $6,04^{\mathrm{ab}} \pm 0,70$ & $3,0^{\mathrm{a}} \pm 0,05$ & $53,31^{\mathrm{g}} \pm 0,49$ \\
\hline PL 36 & $1009^{b c} \pm 75,96$ & $1,22^{\mathrm{b}} \pm 0,19$ & $6,33^{\mathrm{b}} \pm 1,04$ & $5,20^{\mathrm{ab}} \pm 0,63$ & $3,1^{\mathrm{a}} \pm 0,05$ & $62,08^{\mathrm{bcd}} \pm 2,76$ \\
\hline PL 37 & $750^{c} \pm 128,17$ & $0,96^{c} \pm 0,06$ & $6,40^{b} \pm 0,69$ & $6,69^{a} \pm 1,20$ & $2,9^{\mathrm{a}} \pm 0,25$ & $60,16^{\text {cde }} \pm 3,18$ \\
\hline PL 38 & $1044^{\mathrm{bc}} \pm 42,75$ & $1,56^{\mathrm{ab}} \pm 0,38$ & $7,10^{\mathrm{b}} \pm 1,65$ & $3,79^{b} \pm 0,31$ & $3,0^{\mathrm{a}} \pm 0,15$ & $65,41^{\mathrm{b}} \pm 2,35$ \\
\hline PL 39 & $1678^{\mathrm{a}} \pm 245,34$ & $1,67^{\mathrm{ab}} \pm 0,11$ & $11,46^{\mathrm{a}} \pm 1,54$ & $6,68^{a} \pm 0,43$ & $3,1^{\mathrm{a}} \pm 0,20$ & $56,13^{\text {efg }} \pm 1,39$ \\
\hline PL 40 & $1021^{b c} \pm 135,32$ & $1,19^{b c} \pm 0,35$ & $7,26^{\mathrm{b}} \pm 1,36$ & $4,65^{\mathrm{ab}} \pm 0,03$ & $3,2^{\mathrm{a}} \pm 0,11$ & $41,06^{\mathrm{h}} \pm 1,27$ \\
\hline PL 41 & $1293^{\mathrm{abc}} \pm 130,47$ & $1,30^{\mathrm{bc}} \pm 0,25$ & $6,66^{\mathrm{b}} \pm 0,57$ & $5,31^{\mathrm{ab}} \pm 1,49$ & $3,1^{\mathrm{a}} \pm 0,05$ & $62,92^{b c} \pm 1,73$ \\
\hline PL 42 & $1550^{\mathrm{ab}} \pm 348,35$ & $1,59^{\mathrm{ab}} \pm 0,16$ & $7,13^{\mathrm{b}} \pm 0,11$ & $4,49^{\mathrm{ab}} \pm 0,46$ & $3,1^{\mathrm{a}} \pm 0,23$ & $62,79^{b c} \pm 0,35$ \\
\hline PL 43 & $1056^{\mathrm{abc}} \pm 260,10$ & $1,33^{\mathrm{ab}} \pm 0,22$ & $7,16^{b c} \pm 1,25$ & $5,57^{\mathrm{ab}} \pm 1,92$ & $3,2^{\mathrm{a}} \pm 0,41$ & $72,54^{\mathrm{a}} \pm 1,79$ \\
\hline PL 44 & $1222^{\mathrm{abc}} \pm 227,84$ & $1,15^{\mathrm{b}} \pm 0,23$ & $7,73^{b c} \pm 0,46$ & $6,12^{\mathrm{ab}} \pm 1,34$ & $3,5^{\mathrm{a}} \pm 0,43$ & $60,33^{\text {cde }} \pm 1,00$ \\
\hline PL 45 & $1178^{\mathrm{abc}} \pm 88,13$ & $1,11^{\mathrm{b}} \pm 0,06$ & $7,33^{b c} \pm 0,57$ & $5,83^{\mathrm{ab}} \pm 0,67$ & $3,2^{\mathrm{a}} \pm 0,41$ & $58,81^{\text {cdef }} \pm 1,98$ \\
\hline
\end{tabular}


Acerolas destinadas à exportação para a Europa e Japão, devem conter o mínimo de $1000 \mathrm{mg}$ de ácido ascórbico. $100 \mathrm{~g}^{-1}$ de polpa (ALVES, 1996) e, segundo o Instituto Brasileiro de Frutas (1995), o valor de $1200 \mathrm{mg} .100 \mathrm{~g}^{-1}$ é considerado o padrão mínimo exigido pelas indústrias. De acordo com estas exigências, observa-se que todos os genótipos analisados neste trabalho, exceto o PL37, enquadram-se nos requisitos para exportação. No entanto, para a indústria, apenas oito genótipos (PL26, PL28, PL29, PL35, PL39, PL41, PL42, e PL44) apresentaram valores que atendem ao padrão exigido.

A acidez total titulável variou de 0,96 a 1,97 g de ácido málico. $100 \mathrm{~g}^{-1}$ de polpa nos frutos dos genótipos PL37 e PL29, respectivamente (Tabela 1 ), apresentando assim diferença significativa $(\mathrm{p}<0,05)$. Estes valores são semelhantes aos encontrados por vários autores (BRUNINI et al., 2004; MUSSER et al., 2004; NOGUEIRA et al., 2002; GONZAGA NETO; MATTUZ; SANTOS, 1999; ALVES, 1993).

Os ácidos orgânicos contribuem para a acidez e o aroma característico dos frutos. As frutas tropicais, em sua maioria, apresentam uma diminuição nos teores desses ácidos com o amadurecimento, isto devido à utilização destes no ciclo de Krebs, durante o processo respiratório e como fonte de carbono para a síntese de açúcares (KAYS, 1991).

O estado de conservação de frutas pode ser avaliado pela acidez, importante característica em relação ao sabor, juntamente com os valores de sólidos solúveis. Em geral, quando uma fruta passa do estádio de maturação para a senescência, ocorrem várias reações de decomposição, quer sejam por hidrólise, oxidação ou fermentação, alterando assim a concentração dos íons de hidrogênio e, consequentemente, alterando a acidez.

Com relação aos sólidos solúveis totais, os frutos do genótipo PL39 apresentaram o mais elevado valor (11,46 ${ }^{\circ}$ Brix) e os do genótipo PL36, o mais baixo (6,33 $\left.{ }^{\circ} \mathrm{Brix}\right)$ (Tabela 1$)$, apresentando diferença significativa ao nível de $5 \%$ de probabilidade. Segundo Kays (1991), esse aumento é decorrente de processos de biossíntese ou pela degradação de polissacarídeos. A acerola apresenta entre seus açúcares, glicose e frutose e quantidades mínimas de sacarose (SALUNKHE; DESAI, 1984 apud FRANÇA; NARAIN, 2003).

Vários pesquisadores têm encontrado teores de SST para acerolas maduras, variando de 5,7 a 6,5 ${ }^{\circ}$ Brix (BRUNINI et al., 2004); 7,47 a 8,73 ${ }^{\circ}$ Brix (MUSSER et al., 2004); 7,0 a 7,5 ${ }^{\circ}$ Brix (NOGUEIRA et al., 2002) e 4,7 a 9,2 Brix (GONZAGA NETO; MATTUZ; SANTOS, 1999). Segundo Alves (1996), os valores de SST, para acerolas do nordeste, podem variar de 5 até um máximo de $12^{\circ}$ Brix, sendo a média em torno de 7 a $8{ }^{\circ}$ Brix. A chuva ou o uso de irrigação excessiva, na maioria das vezes, reduz o conteúdo de açúcares. As variações numa mesma espécie são decorrentes de fatores diversos como cultivares, tipo de solo, condições climáticas e práticas culturais (CHITARRA, M. I. F.; CHITARRA, A. B., 2005; KAYS, 1991).

De acordo com Alves (1996), as exigências de SST para exportação é de no mínimo 7\% (Europa) ou 7,5\% (Japão). Para a indústria, os valores devem ser acima de $8 \%$, conforme Lopes e Paiva (2002). Sendo assim, os genótipos PL26, PL28, PL29, PL31, PL32, PL33, PL34, PL35, PL38, PL39, PL40, PL42, PL43, PL44 e PL45 atendem ao padrão de exportação e, dentre estes, apenas os genótipos PL29, PL31, PL32, PL34, PL35 e PL39 atingem o padrão de SST exigido pela indústria.
Os Genótipos analisados apresentaram a razão SST/ATT entre 3,79 (PL38) e 7,06 (PL34), com diferença significativa entre elas. A razão SST/ATT determina o sabor dos frutos, uma vez que é a relação entre os açúcares solúveis, isto é, a doçura e a quantidade de ácidos livres presentes nas frutas. Quanto maior for esta razão, mais doces serão as frutas. Sendo assim, pode-se inferir que o genótipo PL 34 caracteriza-se com doçura maior que os demais genótipos. Alves (1993) reporta que, durante a maturação da acerola, a relação SST/ATT aumenta de valores em torno de 4 para aproximadamente 6,5. A determinação da relação açúcares/ácidos em frutas tem como objetivo estabelecer o índice de colheita (KAYS, 1991). França e Narain (2003) relataram valores de SST/ATT para três matrizes de acerola de 4,73 a 9,42, enquanto Musser et al. (2004), em sua pesquisa de caracterização dos primeiros 12 genótipos de aceroleiras cultivadas no BAG da UFRPE, constataram valores de SST/ ATT variando entre 4,27 e 7,31.

Os valores médios de $\mathrm{pH}$ para os dezoito genótipos ficaram entre 2,9 (PL37) e 3,5 (PL44) e não apresentaram diferença significativa como mostra a Tabela 1 . Estes resultados estão dentro da faixa de valores de $\mathrm{pH}$ encontrados por outros autores como França e Narain (2003), cuja variação foi de pH 3,18 a 3,53; Nogueira et al. (2002), que relatou $\mathrm{pH}$ de 3,36 a 3,80; e Musser et al. (2004), pH 3,10 a 3,46. De acordo com Nogueira et al. (2002), com o avanço da maturação, a acerola fica menos ácida, aumentando assim seu $\mathrm{pH}$.

O rendimento de polpa obtido para os dezoito genótipos variou entre 41,06\% (PL40) e 72,54\% (PL43), apresentando diferença significativa entre eles. França e Narain (1998); Alves, Chitarra, A. B.; Chitarra, M. I. F. (1995) encontraram valores de rendimento de polpa de acerola próximos a esta faixa, 56,47 a 63,6\% e 61,33\%, respectivamente. Carpentieri-Pípolo et al. (2002) encontraram rendimento superior (72,1 a 82,55\%) para três novas cultivares de acerola. O rendimento da polpa é uma importante característica na qualidade da acerola destinada ao processamento industrial.

As antocianinas, compostos que pertencem à classe dos flavonoides, são os pigmentos responsáveis pela coloração vermelha da acerola madura, importante aspecto para interesse comercial. Os valores de antocianinas totais dos genótipos caracterizados apresentaram diferença significativa a 5\%, ficando entre 4,35 (PL 26) e 14,93 mg.100 g ${ }^{-1}$ (PL 34), como mostra a Tabela 2. Estes valores estão dentro das médias encontradas por Lima et al. (2003), que foi de 3,79 a 59,74 mg. $100 \mathrm{~g}^{-1}$; por Musser et al. (2004), que relataram valores de 3,8 a 74,40 mg. $100 \mathrm{~g}^{-1}$; e por Mezadri et al. (2008), cujos valores ficaram entre 27,2 e $28,2 \mathrm{mg} .100 \mathrm{~g}^{-1}$. A variação na cor entre cultivares da mesma espécie é usualmente devido às diferenças nas quantidades desses pigmentos (CHITARRA, M. I. F.; CHITARRA, A. B., 2005). Esta coloração também pode variar em função do $\mathrm{pH}$, conforme Bobbio, P. A. e Bobbio, F. (1992).

De acordo com a Tabela 2, pode-se observar que houve diferença significativa entre os valores médios de flavonóis totais, que se encontraram na faixa de 4,40 (PL 26) a $15,04 \mathrm{mg}$ de quercetina.100 $\mathrm{g}^{-1}$ (PL 39). Musser et al. (2004), caracterizando acerolas do BAG/UFRPE, encontraram valores entre 6,73 a 22,22 $\mathrm{mg}$ de quercetina. $100 \mathrm{~g}^{-1}$. 
Tabela 2. Análises de antocianinas e flavonóis totais em frutos de 18 genótipos de aceroleiras do Banco Ativo de Germoplasma da UFRPE.

\begin{tabular}{|c|c|c|}
\hline $\begin{array}{l}\text { Genótipos } \\
\text { (códigos) }\end{array}$ & $\begin{array}{l}\text { Antocianinas totais } \\
\qquad\left(\mathrm{mg} .100 \mathrm{~g}^{-1}\right)\end{array}$ & $\begin{array}{c}\text { Flavonóis totais }^{*} \\
\left(\mathrm{mg} .100 \mathrm{~g}^{-1}\right)\end{array}$ \\
\hline PL 26 & $4,35^{\mathrm{h}} \pm 0,40$ & $4,40^{c} \pm 0,74$ \\
\hline PL 28 & $9,25^{\text {cdef }} \pm 0,25$ & $6,99^{b c} \pm 0,01$ \\
\hline PL 29 & $7,54^{\text {efgh }} \pm 1,15$ & $5,19^{\mathrm{bc}} \pm 0,66$ \\
\hline PL 31 & $8,93^{\text {cdef }} \pm 0,29$ & $8,40^{b c} \pm 0,0$ \\
\hline PL 32 & $6,75^{\mathrm{fgh}} \pm 1,59$ & $8,38^{\mathrm{abc}} \pm 0,84$ \\
\hline PL 33 & $6,84^{\mathrm{fgh}} \pm 1,14$ & $5,75^{\mathrm{c}} \pm 0,74$ \\
\hline PL 34 & $14,93^{\mathrm{a}} \pm 1,55$ & $13,21^{\mathrm{ab}} \pm 0,16$ \\
\hline PL 35 & $12,13^{\mathrm{abc}} \pm 0,45$ & $5,59^{\mathrm{bc}} \pm 0,18$ \\
\hline PL 36 & $13,77^{\mathrm{ab}} \pm 0,29$ & $9,57^{\mathrm{abc}} \pm 0,82$ \\
\hline PL 37 & $8,87^{\mathrm{defg}} \pm 0,25$ & $6,79^{\mathrm{abc}} \pm 0,62$ \\
\hline PL 38 & $9,44^{\text {cdef }} \pm 1,97$ & $9,43^{\mathrm{abc}} \pm 0,15$ \\
\hline PL 39 & $13,80^{\mathrm{ab}} \pm 0,95$ & $15,04^{\mathrm{a}} \pm 0,18$ \\
\hline PL 40 & $6,92^{\text {fgh }} \pm 0,81$ & $9,01^{\mathrm{abc}} \pm 0,65$ \\
\hline PL 41 & $10,88^{\mathrm{bcd}} \pm 1,45$ & $10,20^{\mathrm{abc}} \pm 1,46$ \\
\hline PL 42 & $6,87^{\mathrm{fgh}} \pm 0,02$ & $10,09^{\mathrm{abc}} \pm 0,55$ \\
\hline PL 43 & $5,68^{\mathrm{gh}} \pm 1,09$ & $4,88^{\mathrm{bc}} \pm 0,70$ \\
\hline PL 44 & $6,51^{\text {fgh }} \pm 1,46$ & $5,97^{\mathrm{bc}} \pm 0,07$ \\
\hline PL 45 & $10,64^{\text {bcde }} \pm 0,85$ & $10,80^{\mathrm{abc}} \pm 0,17$ \\
\hline
\end{tabular}

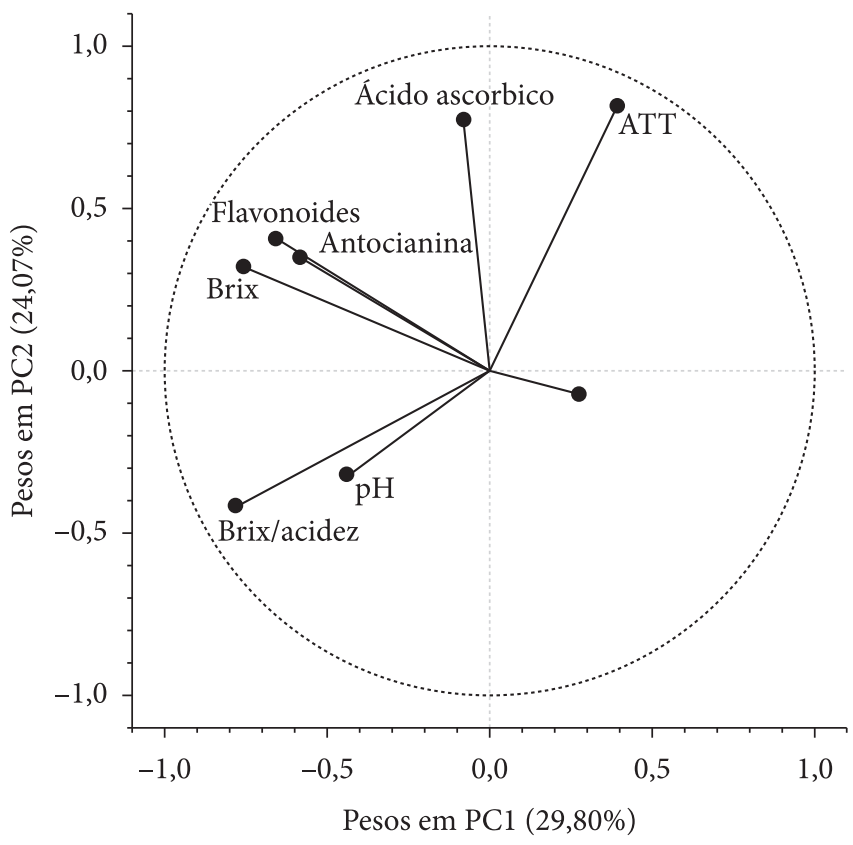

Figura 1. Pesos dos genótipos nas duas primeiras componentes principais para os dados das análises físico-químicas.

Valores entre 5,89 e 20,70 mg de quercetina. $100 \mathrm{~g}^{-1}$ também foram apresentados por Lima et al. (2003), quando avaliaram a estabilidade das antocianinas e dos flavonóis na polpa congelada de acerola. Vendramini e Trugo (2004) encontraram valores de flavonóis de 11,02 e 8,82 mg de quercetina. $100 \mathrm{~g}^{-1} \mathrm{em}$ acerolas das variedades "Flor Branca" e "Okinawa", respectivamente.

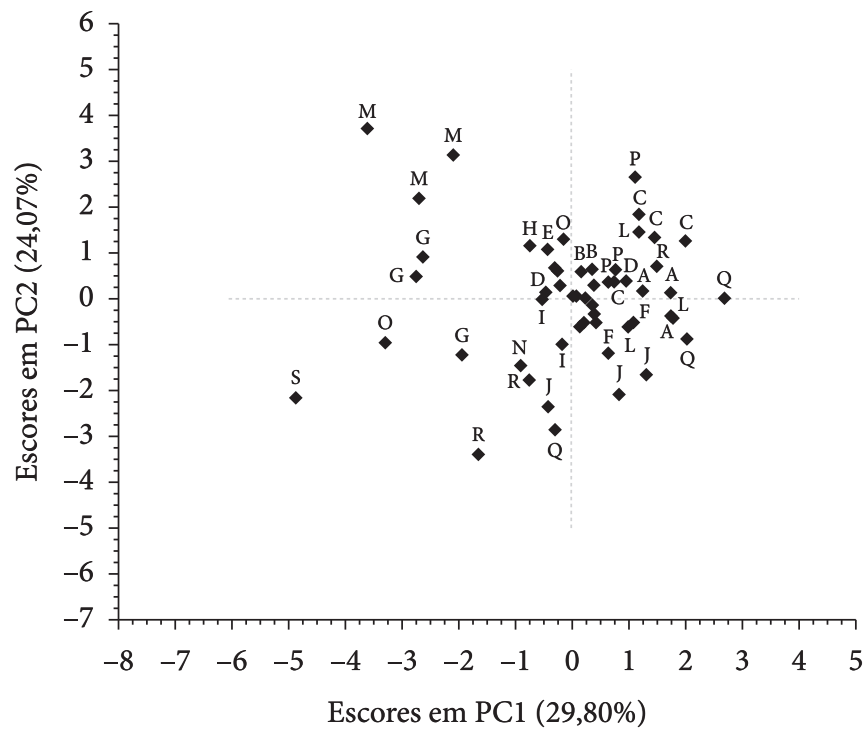

- Active

Figura 2. Escores dos genótipos nas duas primeiras componentes principais para os dados das análises físico-químicas.

O método de análise por componentes principais (PCA) proporcionou um estudo multivariado dos dados experimentais obtidos, facilitando a visualização da correlação entre amostras e variáveis. Os pesos nas duas primeiras componentes principais representam aproximadamente $53,87 \%$ das informações contidas nos valores médios das oito variáveis físico-químicas, identificadas no gráfico com círculos azuis (Figura 1). A primeira componente principal (PC1) reproduz $29,80 \%$ de toda a informação. O peso com valor mais negativo representa a relação -Brix/acidez, ou seja, ao comparar-se com a Figura 2, verifica-se que o genótipo PL 34 (referente à letra S), caracterizou-se por possuir o valor mais alto desta relação.

A segunda componente principal (PC2) corresponde a $24,07 \%$ da informação, com os maiores pesos positivos para AA e ATT, o que destacou o genótipo PL39 (referente à letra M) dos demais, apresentando maiores valores destas variáveis (Figura 2).

\section{Conclusões}

Com exceção do genótipo PL 40, todos os outros apresentaram rendimento acima de $50 \%$ e o genótipo PL 43 se destacou estatisticamente com o maior valor.

Em relação ao teor de ácido ascórbico, todos os genótipos, exceto o PL 37, apresentaram teores superiores a $1000 \mathrm{mg} .100 \mathrm{~g}^{-1}$ de polpa, valor este exigido para exportação. Este mesmo genótipo apresentou, também, o menor valor de ATT e SST. Já o genótipo PL 39 apresentou o maior teor de ácido ascórbico, sólidos solúveis totais e flavonóis, além de elevado teor de antocianinas, sendo, portanto, o mais promissor dentre os dezoito genótipos estudados; enquanto que o PL 34 se destacou por apresentar a maior relação ${ }^{\circ}$ Brix/acidez. 


\section{Agradecimentos}

As autoras agradecem à Universidade Federal Rural de Pernambuco a infraestrutura e à FACEPE/CNPq o auxílio financeiro.

\section{Referências bibliográficas}

AGOSTINI-COSTA, T. S.; ABREU, L. N.; ROSSETI, A. G. Efeito do congelamento e do tempo de estocagem da polpa de acerola sobre o teor de carotenóides. Revista Brasileira de Fruticultura, v. 25, n. 1, p. 56-58, 2003.

ALVES, R. E. Acerola (Malpighia emarginata D. C.): fisiologia da maturação armazenamento refrigerado sob atmosfera ambiente e modificada. 1993. 99 f. Dissertação (Mestrado em Ciência dos Alimentos)-Escola Superior de Agricultura de Lavras, Lavras, 1993.

ALVES, R. E. Características das frutas para exportação. In: GORGATTI NETTO, A. et al. Acerola para exportação: procedimentos de colheita e pós colheita. Brasília: EMBRAPA-SPI, 1996. p. 09-12. (Série Publicações Técnicas Frupex, 21).

ALVES, R. E.; CHITARRA, A. B.; CHITARRA, M. I. F. Postharvest physiology of acerola (Malpighia emarginata D.C.) fruits: maturation changes, respiratory activity and refrigerated storage at ambient and modified atmospheres Acta Horticulturae, v. 370, p. 223-229, 1995.

ASSIS, S. A.; LIMA, D. C.; OLIVEIRA, O. M. M. F. Activity of pectinmethylesterase, pectin content and vitamin $\mathrm{C}$ in acerola fruit at various stages of fruit development. Food Chemistry, v. 74, p. 133-137, 2001.

ASSOCIATION OF OFFICIAL ANALYTICAL CHEMISTS - AOAC. Official methods of analysis. 15th ed. Arlington: 1990.

BOBBIO, P. A.; BOBBIO, F. O. Introdução a química dos alimentos. 2. ed. São Paulo: Varela, 1992.

BRUNINI, M. A. et al. Caracterização física e química de acerolas provenientes de diferentes regiões de cultivo. Revista Brasileira de Fruticultura, v. 26, n. 3, p. 486-489, 2004.

CARPENTIERI-PÍPOLO, V. et al. Novas cultivares de acerola (Malpighia emarginata DC): UEL 3 - Dominga, UEL 4 - Lígia e UEL 5 - Natália. Revista Brasileira de Fruticultura, v. 24, n. 1, p. 124-126, 2002.

Chitarra, M. I. F.; Chitarra, A. B. Pós-colheita de frutos e hortaliças: fisiologia e manuseio. Lavras: UFLA, 2005.

FOUCART, T. Analyse factorielle: programation sur microordinateurs. Paris: Masson, 1982.

FRANÇA, V. C.; NARAIN, N. Caracterização química dos frutos de três matrizes de acerola (Malpighia emarginata). Ciência e Tecnologia de Alimentos, v. 23, n. 2, p. 157-160, 2003.

FRANÇA, V. C.; NARAIN, N. Influência da safra em dois clones de acerola (Malpighia emarginata). In: CONGRESSO BRASILEIRO DE CIÊNCIA E TECNOLOGIA DE ALIMENTOS, 16., 1998, Rio de janeiro Anais... Rio de Janeiro: 1998. CD-ROM.

GONZAGA NETO, L.; MATTUZ, B.; SANTOS, C. A. Caracterização agronômica e clones e aceroleira (Malpighia spp) na região do submédio São Francisco. Revista Brasileira de Fruticultura, v. 21, n. 2, p. 110-115, 1999.
INSTITUTO BRASILEIRO DE FRUTAS - IBRAF. Soluções fruta a fruta: acerola. São Paulo: IBRAF, 1995.

KÄHKÖNEN, M. P.; HEINONEN, M. Antioxidant activity of anthocyanins and their aglycons. Journal of Agricultural and Food Chemistry, v. 51, n. 3, p. 628-633, 2003.

KAYS, S. J. Postharvest physiology of perishable plant products. New York: Van Nostrand Reinhold, 1991.

KRINSKY, N. I. Antioxidant functions of carotenoids. Free Radical Biology \& Medicine, v. 7, p. 617-635, 1989.

LEES, D. H.; FRANCIS, F. J. Standardization of pigment analyses in cranberries. HortScience, v. 7, n. 1, p. 83-84, 1972.

LIMA, V. L. A. G. et al. Avaliação do teor de antocianinas em polpa congelada proveniente de frutos de 12 diferentes aceroleiras (Malpighia emarginata D.C.). Ciência e Tecnologia de Alimentos, v. 23, p. 101-103, 2003.

LIMA, V. L. A. G. et al. Total phenolic and carotenoid contents in acerola genotypes harvested at three ripening stages. Food Chemistry, v. 90, n. 4, p. 495-896, 2005.

LOPES, R.; PAIVA, J. R. Aceroleira. In: BRUCKNER, C. H. (Org.). Melhoramento de fruteiras tropicais. Viçosa: UFV, 2002. v. 1, p. 63-99.

MACIEL, M. I. S. et al. Processing and storage of acerola (Malpighia sp.) fruit and its products. Journal of Food Science and Technology, v. 36, 142-146, 1999.

MARINO NETTO, L. Acerola: a cereja tropical. São Paulo: Nobel; Dieberguer, 1986.

MATSUURA, F. C. A. U.; ROLIM, R. B. Avaliação da adição de suco de acerola em suco de abacaxi visando à produção de um "blend" com alto teor de vitamina C. Revista Brasileira de Fruticultura, v. 24, n. 1, p. 138-141, 2002.

MATSUURA, F. C. A. U. et al Avaliações físico-químicas em frutos de diferentes genótipos de acerola (Malpighia punicifolia L.) Revista Brasileira de Fruticultura, v. 23, n. 3, p. 602-606, 2001.

MEZADRI, T. et al. Antioxidant compounds and antioxidant activity in acerola (Malpighia emarginata DC.) fruits and derivatives. Journal of Food Composition and analysis, v. 21, p. 282-290, 2008.

MUSSER, R. S. et al. Características físico-químicas de acerola do banco ativo de germoplasma em Pernambuco. Ciência e Tecnologia de Alimentos, v. 24, n. 4, p. 556-561, 2004.

NOGUEIRA, R. J. M. C. et al. Efeito do estádio de maturação dos frutos nas características físico-químicas da acerola. Pesquisa Agropecuária Brasileira, v. 37, n. 4, p. 463-470, 2002.

UNIDADES DE PRODUTOS PARA FRUTICULTURA - BASF. Frutas para exportação. Atualidades Agrícolas, n. 6, p. 16-29, 2006.

VENDRAMINI, A. L. A.; TRUGO, L. C. Phenolic compounds in acerola fruit (Malpighia punicifolia, L.). Journal of the Brazilian Chemical Society, v. 15, n. 5, p. 664-668, 2004.

VENDRAMINI, A. L.; TRUGO, L. C. Chemical composition of acerola fruit (Malpighia punicifolia L.) at three stages of maturity. Food Chemistry, v. 71, p. 195-198, 2000.

YAMASHITA, F. et al. Produtos de acerola: estudo da estabilidade de vitamina C. Ciência e Tecnologia de Alimentos, v. 23, n. 1, p. 92-94, 2003. 